\title{
EFFECT OF HOME EXERCISE PROGRAMME ON THIGH MUSCLE STRENGTH BEFORE TOTAL KNEE ARTHROPLASTY
}

\author{
J. Sokk ${ }^{1,3}$, H. Gapeyeva ${ }^{1,3}$, J. Ereline ${ }^{1,3}$, T. Haviko ${ }^{2}$, M. Pääsuke ${ }^{1,3}$ \\ ${ }^{1}$ Institute of Exercise Biology and Physiotherapy, University of Tartu, \\ Tartu, Estonia \\ ${ }^{2}$ Department of Orthopaedics and Traumatology, \\ Tartu University Hospital, Tartu, Estonia \\ ${ }^{3}$ Centre of Behavioural and Health Sciences, \\ University of Tartu, Tartu, Estonia
}

\begin{abstract}
The aim of the study was to assess the effect of home exercise programme (HEP) on thigh muscle strength in patients with knee osteoarthritis (OA) before unilateral total knee arthroplasty (TKA).

Five femaleOA patients with mean age $67.8 \pm 5.9$ years participated in the study. Isometric maximal voluntary contraction (MVC) force of the quadriceps femoris (QF) and hamstring (HM) muscles was measured by hand-held dynamometry. Knee active range of motion (aROM) in flexion was measured by goniometer. Knee pain score was evaluated by knee pain scale before and after HEP.

A significant reduction $(p<0.05)$ in MVC force of the QF and HM muscles and aROM in flexion were noted before HEP, comparing the involved and uninvolved leg. After HEP, MVC force of the QF muscle increased significantly $(p<0.05)$ for the involved and uninvolved leg, whereas no significant differences $(p>0.05)$ were observed between the limbs. MVC force of the HM muscle and aROM in flexion remained significantly lower $(p<0.05)$ for the involved leg as compared with the uninvolved leg after HEP. Knee pain score was significantly $(p<0.05)$ higher for the involved leg before and after HEP as compared with the uninvolved leg.
\end{abstract}


Two months before the operation, HEP was an effective therapeutic procedure for increasing $\mathrm{QF}$ muscle strength in patients who underwent TKA.

Key words: osteoarthritis, home exercise programme, isometric maximal voluntary contraction force, aROM

\section{INTRODUCTION}

Osteoarthritis (OA) is one of the most common chronic degenerative joint disorders and the prevalence increases with age throughout the elderly years [5]. Patients with OA have the pain that typically worsens with weight bearing and activity and improves with rest, as well as morning stiffness and swelling of the involved joint after periods of inactivity. On physical examination, they often have tenderness on palpation, bony enlargement, crepitus on motion, and/or limitation of joint motion [15]. Stefanic et al. [22] have demonstrated that the prevalence of lateral and medial patellofemoral joint cartilage damage and bone marrow lesions was higher in weaker compared to stronger knees. Ikeda et al. [7] suggest the possibility that age-related quadriceps-dominant muscle atrophy can play a role in the pathogenesis of knee OA. Segal et al. [19] concluded that in women but not in men, quadriceps muscle weakness appears to increase the risk of joint space narrowing over 30 months in the Multicenter Osteoarthritis study. Bade et al. [1] measured changes in muscle strength, range of motion, and function from 2 weeks before to 6 months after total knee arthroplasty (TKA) and compared them with data from healthy older adult control group. They found that patients performed significantly worse at all times for all measures as compared to healthy older adults. Mizner et al. [12] hypothesized in their study that preoperative quadriceps muscle strength is the best predictor of postoperative functional ability, compared to preoperative pain or knee range of motion (ROM). They concluded that preoperative quadriceps muscle strength plays a dominant role in predicting one-year Stair Climbing Test (SCT) and Timed Up and Go Test (TUG) functional measures, but it is not a good predictor of the score on self-report questionnaires. Preoperative body pain and knee flexion ROM are poor predictors of all functional outcome measures. Pertella [14] asked in her review article whether exercise is an effective treatment for osteoarthritis of 
the knee, and found that exercises had short-term effects in the treatment of patients with OA. Rodgers et al. [17] compared preoperative physiotherapy group with controls. The assessment included: Hospital for Special Surgery knee rating scale, knee range of motion, thigh circumference, walking speed, isokinetic knee flexion and extension testing, and computed tomography scanning for crosssectional muscle area. They found that this study failed to support the routine use of preoperative physical therapy in knee replacement surgery. Jaggers et al. [9] investigated 4-week pre-rehabilitation in two women who underwent TKA. The functional outcome included a 6-minute walk test, the number of times of getting up from a chair in $30 \mathrm{~s}$, proprioception, and self-reported function and pain estimation using the Western Ontario and McMaster University Osteoarthritis Index. They concluded that 4-week pre-rehabilitation had a positive effect on functional task performance and knee proprioception before surgery.

The aim of the current study was to assess the effect of home exercise programme in patients with knee OA on thigh muscle strength before unilateral total knee arthroplasty.

It was hypothezised that isometric maximal voluntary contraction (MVC) force of the QF and HM muscles, and aROM in flexion can be improved in patients with knee OA after 2-month preoperative therapeutic exercise programme, conducted at home.

\section{METHODS}

\section{Subjects}

Five female patients with OA aged 59 to 74 years undergoing TKA for III-IV stage knee OA participated in this study. The mean \pm SE age of the patients was $67.8 \pm 2.7$ years, height $159.4 \pm 2.5 \mathrm{~cm}$, body mass $74.7 \pm 5.8 \mathrm{~kg}$, and BMI $29.4 \pm 1.9 \mathrm{~kg} / \mathrm{m}^{2}$. The subjects were recruited by orthopedic surgeons in the Department of Orthopaedics and Traumatology of Tartu University Hospital, between February and June 2011. The exclusion criteria included inflammatory arthritis, obesity $\left(\mathrm{BMI}>30 \mathrm{~kg} / \mathrm{m}^{2}\right)$, implantated pacemakers, progressive neurological disease, diabetes, head injuries, cardiological diseases NYHA III-IV, osteoporosis, deep vein thrombosis, COPD. The study carried the approval of the Tartu University Ethics Committee. 


\section{Home exercise programme (HEP)}

The preoperative therapeutic HEP was mainly oriented to muscle strengthening exercises with elastic band (Thera-Band, System of Progressive Exercise, US), and stretching, balance, and walking exercises. At the first meeting the physiotherapist taught the exercise programme to the patients, gave them the elastic band set, the printed exercise programme with pictures and explanations, and diary. The patients recorded every day several data in their diary - for how many times they did each exercise, the training time, perception of pain before and after HEP, time spent in outdoor walking, and the number of stairs walked during the day. Once a week the physiotherapist called the patients on the phone, and asked them about HEP and problems with the knee. The patients performed the exercises for 64 days on the average, whereas one patient did the exercises twice a day.

\section{Isometric maximal voluntary contraction force}

Isometric MVC force of the QF and HM muscles was measured by hand-held dynamometer (Lafayette Manual Muscle Test System). During QF muscle isometric strength testing the subject was in the seated position on an investigation bed with knee and hip flexed to 90 deg. Hand-held dynamometer was placed proximal to the ankle on anterior surface of leg. During HM muscle isometric strength testing, the subject was in the prone position. Hand-held dynamometer was placed proximal to the ankle on posterior surface of leg $[2,10]$. The positions were supervised by the investigator and the subjects were encouraged to act in the desired way. The subjects were required to exert isometric MVC force pushing against the dynamometer for approximately $3 \mathrm{~s}$ before each contraction, and instructed to "push as hard as possible". The best results from 3 attempts were taken as isometric MVC force. A rest period of $1 \mathrm{~min}$ was allowed between attempts.

Stark et al. [21] wrote a systematic review on hand-held dynamometry correlation with gold standard isokinetic dynamometry and found that the research results demonstrated minimal differences between hand-held dynamometry and isokinetic testing.

\section{Knee flexion range of motion measurement}

Knee flexion aROM was collected in prone position using the standard long arm goniometer. The axis of the goniometer was placed 
over the lateral femoral epicondyle. The proximal end of the goniometer was aligned with the great trochanter of the femur and the distal arm was aligned with the lateral malleous. During knee flexion aROM measurement, the subjects were instructed to maximally flex the knee and draw the heel toward the buttocks. The average peak knee flexion was recorded from three trials. Goniometric knee flexion measurements are highly reliable in persons with knee OA [4].

\section{Pain assessment}

Self-assessment of knee pain in everyday activities was provided by reports on 10-point visual analogue scale (VAS) with endpoints of "no pain" (0) and "worst possible pain" (10) [16]. The mean point score in five different activities was calculated for pain in both legs perceived during resting, walking, walking upstairs and downstairs, and during the effort.

\section{Statistical analysis}

Data are mean and standard error $( \pm \mathrm{SE})$. One-way analysis of variance (ANOVA) followed by Turkey post hoc comparisons was used to evaluate differences between the involved and uninvolved leg. A paired t-test was used to evaluate differences between pre- and postHEP session. A level of $\mathrm{p}<0.05$ was selected to indicate statistical significance.

\section{RESULTS}

Before HEP, patients with knee OA showed a significant reduction $(p<0.05)$ in the MVC force of the QF and HM muscles for the involved leg as compared with the uninvolved leg (Figure 1). After HEP, MVC force of the QF muscle increased $(\mathrm{p}<0.05)$ as compared with the pre-exercising level for the involved and uninvolved leg (Figure 1a). MVC force of the QF muscle in patients with knee OA did not differ significantly $(\mathrm{p}>0.05)$ as compared with the uninvolved leg, whereas the HM muscle MVC force remained significantly lower $(p<0.05)$ after HEP as compared with the uninvolved leg (Figure 1b). 

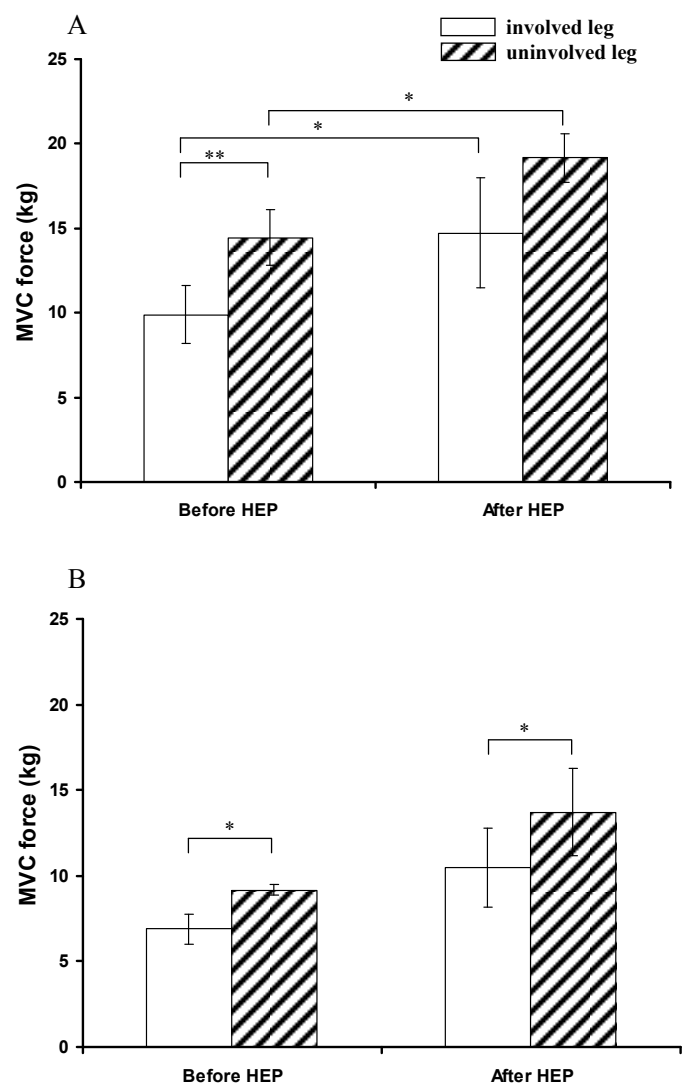

Figure 1. Mean $( \pm \mathrm{SE})$ quadriceps femoris $(\mathrm{QF})(\mathrm{A})$ and hamstring $(\mathrm{HM})$ muscle groups (B) isometric maximal voluntary contraction (MVC) force in patients with knee OA before and two months after home exercise programme (HEP) $(\mathrm{n}=5),{ }^{*} \mathrm{p}<0.05, * * \mathrm{p}<0.001$.

Before HEP, patients with knee OA showed a significant reduction $(p<0.05)$ in the aROM in flexion for the involved leg as compared with the uninvolved leg. (Figure 2). After HEP, aROM in flexion increased for the uninvolved leg $(\mathrm{p}<0.05)$ as compared with the preexercising level (Figure 2a.), whereas the involved leg aROM in flexion remained significantly lower $(\mathrm{p}<0.05)$ after HEP as compared with the uninvolved leg. 

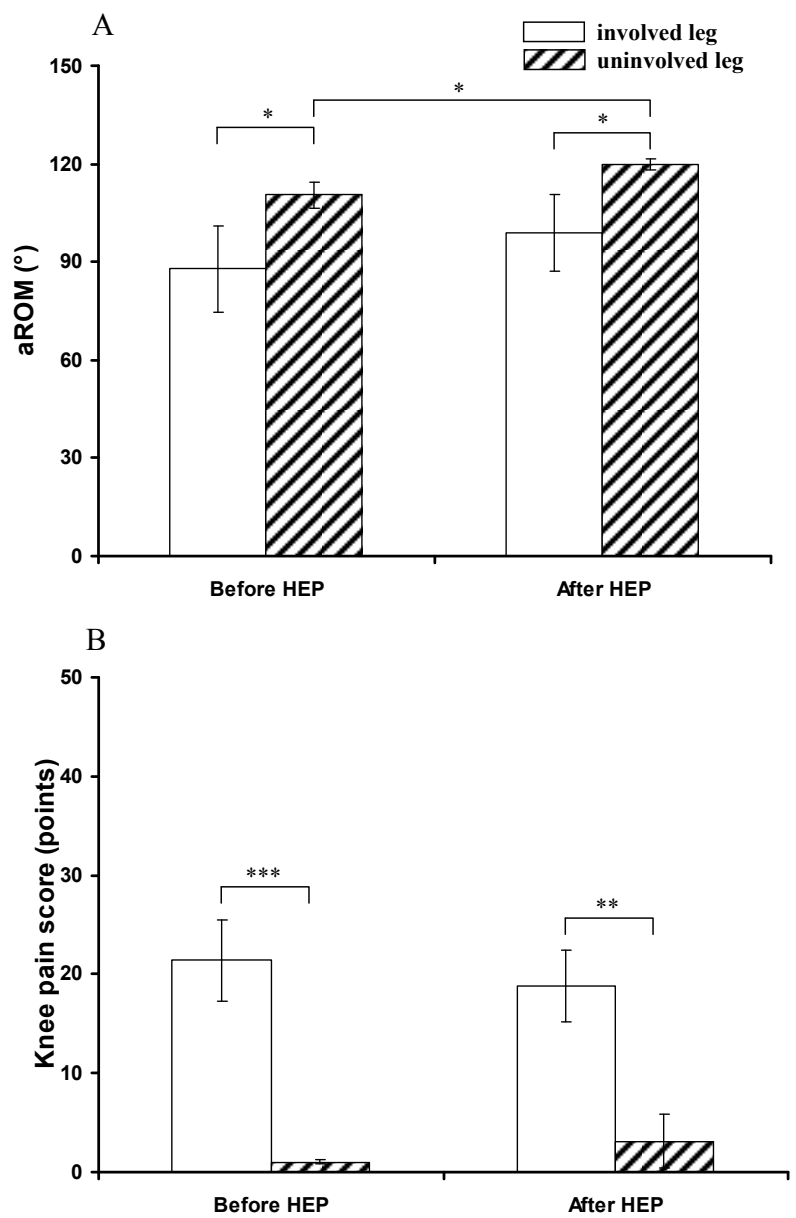

Figure 2. Mean $( \pm \mathrm{SE})$ knee active range of motion (aROM) in flexion (A) and pain assessed by 10-point visual analogue scale (B) in patients with knee $\mathrm{OA}$ before and two months after home exercise programme (HEP) $(\mathrm{n}=5),{ }^{*} \mathrm{p}<0.05, * * \mathrm{p}<0.01, * * * \mathrm{p}<0.001$.

Knee pain score points for the involved leg were significantly $(p<0.05)$ higher before HEP as compared with the uninvolved leg, and remained higher also after HEP (Figure 2b). 


\section{DISCUSSION}

This study evaluated the effect of 2-month HEP on thigh muscle strength and aROM in patients with knee OA before TKA. The main finding of the study was the increase of MVC force in the QF muscle after 2-month HEP. Our results indicated that before HEP the MVC force of the QF and HM muscles for the involved leg was 32 and 25\% lower respectively, as compared with the uninvolved leg. After 2-month HEP, MVC force of the QF and HM muscle for the involved leg increased 32 and $34 \%$ respectively as compared to the pre-HEP level, whereas there were no significant differences in $\mathrm{QF}$ muscle MVC force between the limbs. MVC force of HM muscles for the involved leg remained significantly lower two months after HEP as compared to the uninvolved leg. Patient No1 had 60 HEP sessions, and patient No 2 had 48 sessions - however, she performed exercises twice in the day, patient No 3 had 67 exercise sessions, patient No 4 had 67 sessions and patient No 5 had 88 sessions. One session lasted for 20-40 minutes. Swank et al. [24] compared patients with severe OA who underwent TKA. One group had usual care, the other group usual care and exercises that included resistance training using bands, flexibility, and step training at least three times per week for 48 weeks before TKA. They found that in the exercise group increased the leg strength (isokinetic peak torque for knee extensors and flexors), and the ability to perform functional tasks. They concluded that short-term (4-8 weeks) prerehabilitation was effective, easily transferred to a home environment, and they proposed that clinicians consider prerehabilitation before TKA. Brown et al. [3] in their case study measured isokinetic knee extension (QF) and flexion (hamstrings) with Biodex Isokinetic Dynamometer. A patient had prerehabilitation 4 weeks before TKA. After prerehabilitation, the patient demonstrated a 54\% strength gain in her extension and a $34 \%$ gain of flexion strength of her involved leg. The prerehabilitation intervention emphasized three components: resistance training, flexibility, and step training; three session's treatment per week for four weeks. Rooks et al. [18] investigated the effect of a short preoperative exercise intervention on the functional status, pain and muscle strength of patients before and after total knee joint arthroplasty. They used the 1-repetition maximum leg press test to determine lower-extremity strength. The patients' baseline leg strength was $97 \pm 37 \mathrm{~kg}$, and the preoperative level was $116 \pm 37 \mathrm{~kg}$. The 
patients performed water and land-based exercises three times weekly over a 6-week period prior to surgery. The result of the studies showed that after prerehabilitation in clinics, the involved leg muscles strength was increased. In our study, patients were instructed to perform the home exercise programme on the first meeting and after that they performed the exercises at home. Physiotherapist called on the phone to the patients every week and asked about the training results. Home exercise programme is a cheapest way to improve patients' condition before TKA. However, two patients canceled the surgery. The improvement of $\mathrm{QF}$ muscle isometric force-generation capacity after 2-month HEP can be explained by neural adaptation mechanisms. It has been suggested that exercise training for less than 2-3 months does not induce a significant hypertrophy in skeletal muscles [13]. Speed and Campbell [20] found that significant gains in strength in individuals with rheumatoid arthritis could occur due to neural adaptation without gains in muscle mass during the strengthening programme.

In our study, knee aROM in flexion before HEP for the involved leg was $21 \%$ lower as compared with the uninvolved leg. After HEP, the knee aROM in flexion for the involved leg increased $11 \%$. Ipach et al. [8] reported that in patients before total knee arthroplasty mean knee aROM in flexion was $94 \mathrm{deg}$. Li et al. [11] found that preoperative knee aROM in flexion was $95 \mathrm{deg}$. Stratford et al. [23] showed in their study that pROM in flexion was $109 \mathrm{deg}$. These data are similar to the results obtained in our study. The significant knee aROM increase before TKA is difficult expect in this group of patients, because OA induced changes in the knee joint and surrounding tissues. All tissues of the joint are involved, including bone, synovium, muscles, nerves and ligaments [6]. One limitation factor of this study was a small sample size (5 patients).

The pain measured by knee pain score before HEP was reduced $17 \%$ after HEP. Before HEP the uninvolved leg of the patients was pain free, whereas after HEP one patient reported pain in both legs. Knee pain was worse during stair walking, especially going downstairs, and in the activities when patients carried extra weight in their hands (shopping). Rooks et al. [18] and Walls et al. [25] reported in their studies that the involved knee pain had a tendency to decrease.

In conclusion, this study demonstrated that 2-month HEP was effective for increasing QF muscle isometric strength in patients with OA. Isometric strength of HM muscles and knee aROM in flexion had 
a tendency to increase and knee pain for the involved leg had a tendency to decrease after HEP. It can be recommended to use home exercises (including mobility, strength and aerobic exercises) in the last stage of knee OA before TKA.

\section{REFERENCES}

1. Bade M. J., Kohrt W. M., Stevens-Lapsley J. F.(2010) Outcome before and after total knee arthroplasty compared to healthy adults. J. Orthop. Sports Phys. Ther. 40: 559-567

2. Bohannon R. W. (1986) Test-retest reliability of hand-held dynamometry during single session of strength assessment. Phys. Ther. 66: 206-209

3. Brown K., Swank A. M., Queasada P. M., Nyland J., Malkani A., Topp R. (2010) Prehabilitation versus usual care before total knee arthroplasty: a case report comparing outcomes with the same individual. Physiother. Theory Pract. 26: 399-407

4. Gogia P. P., Braatz J. H., Rose S. J., Norton B. J. (1987) Reliability and validity of goniometric measurements at the knee. Phys. Ther. 67: $192-195$

5. Felson D. T., Naimark A., Anderson J., Kazis L., Casterlli W., Meenan R. F. (1987) The prevalence of knee osteoarthritis in the elderly. The Framingham Osteoarthritis Study. Arthritis Rheum. 30: 914-918

6. Goldring M. B., Goldring S. R. (2010) Articular cartilage and subchondral bone in the pathogenesis of osteoarthritis. Ann. NY Acad. Sci. 1192: 230-237

7. Ikeda S., Tsumura H., Torisu T. (2005) Age-related quadricepsdominant muscle atrophy and incident radiographic knee osteoarthritis. J. Orthop. Sci. 10: 121-126

8. Ipach I., Mittag F., Lahrmann J., Kunze B., Kluba T. (2011) Arthrofibrosis after TKA - influence factors on the absolute and gain in flexion after manipulation under anaesthesia. BMC Musculoskelet. Disord. 12: 184

9. Jaggers J. R., Simpson C. D., Frost K. L., Quesada P. M., Topp R. V., Swank A. M., Nyland J. A. (2007) Prerehabilitation before knee arthroplasty increases postsurgical function: a case study. J. Strength Cond. Res. 21: 632-634

10. Knols R. H., Audemkampe G., de Bruin E. D., Uebelhart D., Aaronson N. K. (2009) Hand-held dynamometry in patients with haematological malignancies: measurement error in the clinical 
assessment of knee extension strength. BMC Musculoskelet. Disord. 10: 31

11. Li B., Wen Y., Liu D., Tian L. (2011) The effect of knee postition on blood loss and range of motion following total knee arthroplasty. Knee Surg. Sports Traumatol. Arthrosc. (Epub ahead of print).

12. Mizner R. L., Petterson S. C., Snyder-Macer L. (2005) Quadriceps strength and the time course of functional recovery after total knee arthroplasty. Orthop. Sports Phys. Ther. 35: 424-436

13. Moritani T., de Vries H. A. (1979) Neural factors versus hypertrophy in the time course of muscle strength gain. Am. J. Phys. Med. 58: $115-130$

14. Pertella R. J. (2000) Is exercise effective treatment for osteoarthritis of the knee? Br. J. Sports Med. 34: 326-331

15. Recommendations for the medical management of osteoarthristis of the hip and knee: 2000 update (2000) American College of Rheumatology Subcommittee on Osteoarthritis Guidelines. 43: 1905-1915

16. Rejeski W. J., Ettinger W. H. Jr., Shumaker S., Heuser M. D., James P., Monu J., Burns R. (1995) The evaluation of pain in patients with knee osteoarthritis: the knee pain scale. J. Rheumatol. 22: 1124-1129

17. Rodgers J. A., Garvin K. L., Walker C. W., Morford D., Urban J., Bedard J. (1998) Preoperative physical therapy in primary total knee arthroplasty. J. Arthroplasty. 13: 414-412

18. Rooks D. S., Huang J., Bierbaum B. E., Bolus S. A., Rubano J., Connolly C. E., Alpert S., Iversen M. D., Katz J. N. (2006) Effects of preoperative exercise on measures of functional status in men and women undergoing total hip and knee arthroplasty. Arthritis. Rheum. 55: 700-708

19. Segal N. A., Glaus N. A., Torner J., Yang M., Felson D. T., Sharma L., Nevitt M., Lewis C. E. (2010) Quadriceps weakness predicts risk for knee joint space narrowing in women in the MOST cohort. Osteoarthritis Cartilage. 18: 769-775

20. Speed C. A., Campbell R. (2010) Mechanism of strength gain in handgrip exercise programme in rheumatoid arthritis. Rheumatol Int. (Epub ahead of print)

21. Stark T., Walker B., Philips JK., Fejer R., Beck R. (2011) Hand-held dynamometry correlation with the gold standard isokinetic dynamometry: a systematic review. PM R. 3: 472-479

22. Stefanic J. J.,Guermazi A., Zhu Y., Zumwalt A. C., Gross K. D., Clancy M., Lynch J. A., Segal N. A., Lewis C. E., Roemer F. W., Powers C. M., Feldson D. T. (2011) Quadriceps weakness, patella alta and, structural features of patellofemoral osteoarthritis: the multicenter osteoarthritis study. Arthritis Care Res. 63: 1391-1397 
23. Stratford P. W., Kennedy D. M., Robarts S. F. (2010) Modelling knee range motion post arthroplasty: clinical applications. Physiother. Can. 62: $378-387$

24. Swank A. M., Kachelman J. B., Bibeau W., Quesada P. M., Malkani A. Tropp R. V. (2011) Prehabilitation before total knee arthroplasty increases strength and function in older adults with severe osteoarthritis. J. Strength Cond. Res. 25: 318-325

25. Walls J. R., McHugh G., O’Gorman D. J., Moyna N. M., O’Byrne J. M. (2010) Effects of preoperative neuromuscular electrical stimulation on quadriceps strength and functional recovery in total knee arthroplasty. A pilot study. BMC Musculoskelet. Disord. 11: 119.

\section{ACKNOWLEDGEMENTS}

This study was supported by Estonian Ministry of Education and Research, project No SF0180030s07, Estonian Science Foundation project No 7939, and by European Union FP7 223576 project.

\section{Correspondence to:}

Jelena Sokk

Institute of Exercise Biology and Physiotherapy,

University of Tartu

Jakobi 5, 51014, Tartu

Estonia

E-mail: jelena.sokk@ut.ee 